RESEARCH PAPER

\title{
Stimulants of tea Infusion are Completely Withdrawn by SCOBY during Kombucha Fermentation: A Biochemical Investigation
}

\author{
Soumya Majumder, Arindam Ghosh, Sourav Chakraborty and \\ Malay Bhattacharya* \\ Molecular Biology and Tissue Culture Laboratory, Department of Tea Science, University of North Bengal, Siliguri, \\ Darjeeling, India \\ *Corresponding author: malaytsnbu@gmail.com
}

Paper No.: 249

Received: 05-10-2020

Revised: 25-11-2020

Accepted: $17-12-2020$

\begin{abstract}
Caffeine content in kombucha is very much lower than that of normal tea infusion. But, this stimulant plays an important role in kombucha fermentation as it regulates the starter or SCOBY (symbiotic colony of bacteria and yeasts) to produce the cellulose network on broth to accelerate the fermentation process. This research was designed to investigate the aftermath of caffeine and related tea alkaloids in kombucha through preliminary biochemical tests and chromatographic analysis where both the broth (beverage) and SCOBY (the cellulose layer) were taken as individual samples. The beverage clearly replied negative in all the tests where the SCOBY extract showed richness in alkaloid content. Moreover, GC-MS analysis revealed presence of caffeine $(8.7 \%)$; guanosine $(12.01 \%)$, the precursor of caffeine; thymine $(4.08 \%)$; and some undesirable components which has confirmed that SCOBY has an ability to capture a huge amount of stimulants from tea during fermentation.
\end{abstract}

Keywords: Caffeine, stimulants, tea, kombucha, SCOBY

It is widely accepted that tea leaf and its infusion contain caffeine and other stimulants or alkaloids in significant quantities. Consequently it is also reported that, these stimulants (especially caffeine) are either absent or found in a very low quantity in the probiotic fermented version of tea beverage, called kombucha. According to Malbaša et al. (2006), quantity of these alkaloids gets decreased during fermentation of tea to kombucha. Interestingly, reports have confirmed that caffeine and other stimulants can stimulate the ability of Acetobacter xylinum (primary bacterium of SCOBY) to produce a cellulose network, the base of kombucha's microflora (Fontana et al. 1991). According to Greenwalt et al.
(2000), methylxanthines and other stimulants can also inhibit the normal switch-off mechanism of cellulose synthase which may also help to accelerate the formation of that cellulose network. However, there are no report that can clarify the existence of those stimulants in kombucha; whether as by-products of microbial metabolism during fermentation or its involvement into some other microbial pathways inside the SCOBY. This throws a doubt on the fate of caffeine and other alkaloids of tea infusion in its

How to cite this article: Majumder, S., Ghosh, A., Chakraborty, S. and Bhattacharya, M. (2020). Stimulants of tea Infusion are Completely Withdrawn by SCOBY during Kombucha Fermentation: A Biochemica Investigation. Int. J. Food Ferment. Technol., 10(2): 113-118.

Source of Support: None; Conflict of Interest: None क क 
fermented form. So, to find out the aftermath of those components in kombucha, we prepared and carried on preliminary biochemical tests, followed by GCMS analysis to put up a scientific explanation for better understanding.

\section{MATERIALS AND METHODS}

\section{Preparation of samples}

A strong tea infusion was prepared with CTC tea (1\% $\mathrm{w} / \mathrm{v}$ ) for fifteen minutes so as to obtain alkaloids in the infusion as much as possible. Sucrose $(6 \% \mathrm{w} / \mathrm{v})$ was used as nutrient for the microbial culture inside the tea broth. Following protocol of Greenwalt et al. (2000) and Zhu et al. (2014), a number of kombucha batches were prepared and incubated under a sterile condition. Healthy batch of kombucha was selected after 30 days of incubation. The broth or kombucha $(\mathrm{KB})$ and the thick floating SCOBY were taken out separately as different samples source for further experiments. For obtaining extract, SCOBY was squeezed hard to separate SCOBY extract (SE) and the dry and hard remaining was discarded (Fig. 1 a, $b, c)$.

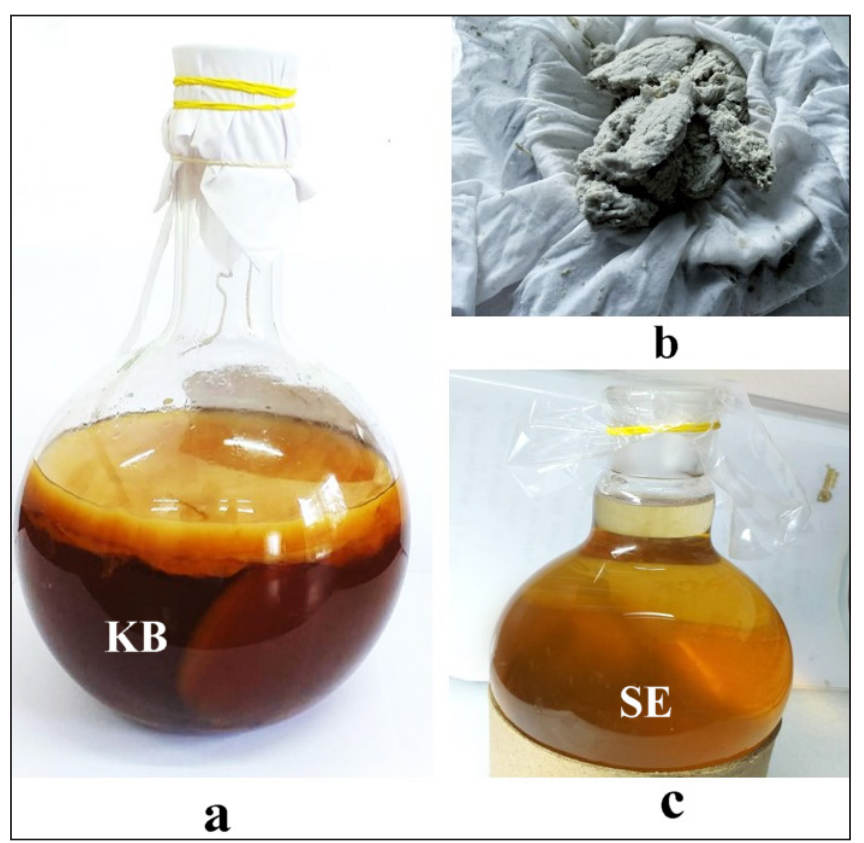

Fig. 1. Image of (a) selected broth containing SCOBY, (b) squeezed cellulose and (c) SCOBY extract

\section{Preliminary biochemical tests}

Tests were performed with both KB and SE to detect alkaloids and caffeine. Alkaloids were tested by Mayer's, Wagner's and Marme's tests (Santra et al. 2006) where formation of precipitate confirms the presence of alkaloids. Caffeine detection was carried on following murexide test (Santra et al. 2006) where appearance of purple colour confirms the presence of caffeine.

Following the technique of column chromatography (Bhattcharya et al. 2009), we separated caffeine and related alkaloids from both $\mathrm{KB}$ and SE through a silica gel (200-400 mesh size) packed column. As analyte, $10 \mathrm{ml}$ of each $\mathrm{KB}$ and SE were used and for mobile phase, two solvents (chloroform and dicholoromethane) were selected on the basis on affinity of targeted compounds towards solvents. Each fractions were collected individually, dried and tested for caffeine confirmatory test (the murexide test).

\section{Gas chromatography mass spectrometry analysis}

$\mathrm{KB}$ and SE (1 ml each) were taken in individual test tubes and left for complete air drying. The dried samples were finally dissolved in $1 \mathrm{ml}$ of ethanol prior to GC-MS analysis. The analysis was performed by GCMS-QP2010 Plus (Shimadzu Corporation, Kyoto Japan) attached with a Rxi-5 fused-silica capillary column $(0.25 \mu \mathrm{m}$ film thickness, $0.25 \mathrm{~mm}$ internal diameter and $30 \mathrm{~m}$ of length) following the protocol of Majumder et al. (2020).

\section{RESULTS AND DISCUSSION}

\section{Preliminary biochemical tests}

Results of preliminary tests have supported previous reports as the beverage or $\mathrm{KB}$ resulted negative in all the alkaloid detection while biofilm extract or SE showed positive result. Moreover, the murexide test for caffeine detection also gave a positive result for SE only. These results clearly indicates that SE contains the alkaloids of tea and one of their major components caffeine unlike the komucha (KB). 
In column chromatographic separation of caffeine and related components, chloroform and dicholoromethane were used as mobile phase because our targeted compounds are already reported to be highly soluble in those two organic solvents (JaberVazdekis et al. 2006; Shalmashi and Golmohammad, 2010). Presence of caffeine was simply recognized as the fractions showed white crystalline structures. Furthermore, the murexide test was again assessed to confirm presence of caffeine and related compounds and to check whether KB contains any traces of such compound or not. But, the murexide test also resulted negative for KB.

Meanwhile, results of the above experiments already confirmed that sample SE contains caffeine and related alkaloids in it unlike the beverage. These result have not only proven that SCOBY needs caffeine and other stimulants for growing and stimulating the fermentation process but also suggested that alkaloids or stimulants (like caffeine), a major part of tea may get removed from the infusion as SCOBY picks all of them during kombucha fermentation. Now, to evaluate and judge the results of these tests, GC-MS study was conducted.

\section{Gas chromatography mass spectrometry analysis}

GC-MS chromatograms are given in Fig. 2 and peak reports of both KB and SEare represented in Table 1 and Table 2 respectively. Expectedly, SCOBY extract (SE) showed richness in caffeine content with an amount of $8.70 \%$ while, in the beverage or $\mathrm{KB}$, there was no traces of caffeine and related compounds. Moreover, besides caffeine, another alkaloid and major purine nucleotide of tea, guanosine (as guanosine hydrate) was detected as the major compound of SE with a peak area of $12.01 \%$. Interestingly, the compound guanosine is also known to be present in tea leaves and that too as a precursor of caffeine (Negishi et al. 1992). Now, if we accept the fact that SCOBY has captured all the stimulants from broth, then presence of another SE component; e.g. thymine $(4.08 \%)$, a pyrimidine plant stimulant (Šormová et al. 1960), should also be taken into consideration along with caffeine and guanosine. However, in $\mathrm{KB}$, there were neither any traces of above mentioned compounds nor any other stimulants. So, this is definitely a remarkable confirmation to our interpretation that microflora of SCOBY biofilm has picked up all the tea stimulants from kombucha which have been detected by GC-MS (Fig. 3). Besides alkaloids, presence of an infamous flaw compound of wines, isovaleric acid $(5.45 \%) ;$ a toxic compound, 1,3-Dioxolane, 4-methylene- or DABCO (1.96\%) and a susceptible oxidative agent, 1,3-dioxolane, 4-methylene $(2.09 \%)$ in SE is also a good sign as these substandard and unacceptable components were completely absent in $\mathrm{KB}$ or might have been removed by the biofilm in the same way like the stimulants. However, isovaleric acid is also a reported metabolite of Brettanomyces sp. (Licker et al. 1998), a common yeast found in SCOBY (Greenwalt et al. 2000).

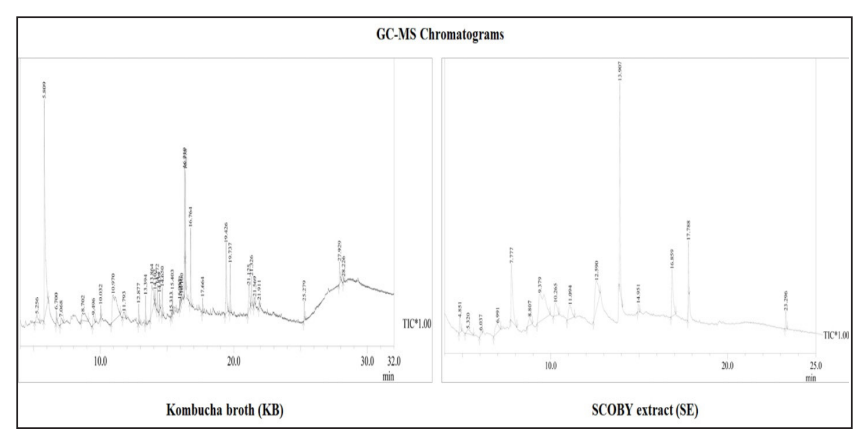

Fig. 2: GC-MS chromatograms

The GC-MS analysis and other biochemical tests have helped to discover that both samples, the beverage and the biofilm extract, may be a part of the same broth, but, are distinct to each other regarding their chemical characters. Moreover, the secret chemical profile has also been unfolded for SCOBY extract through this set of experiments. It can be assumed that Acetobacter xylinum is one of them as it has previously been reported to produce the cellulose network stimulated by caffeine and other stimulants (Fontana et al. 1991; Greenwalt et al. 2000). But, through a biochemical analysis, it is very hard to identify responsible and specific organism(s) involved in picking up those stimulants. Moreover, caffeine is also known to exhibit antimicrobial activity. So, chances may be less for other microorganisms to capture this big 
Table 1: GC-MS peak report of kombucha beverage (KB)

\begin{tabular}{|c|c|c|c|c|}
\hline Peak\# & R.Time & Area & Area\% & Compounds of KB \\
\hline 1 & 5.256 & 205343 & 1.9 & Acetamide, N,N'-carbonylbis- \\
\hline 2 & 5.809 & 2847707 & 26.41 & 2-Cyclopenten-1-one, 2-hydroxy- \\
\hline 3 & 6.7 & 80876 & 0.75 & 2,4-Dihydroxy-2,5-dimethyl-3(2H)-furan-3-one \\
\hline 4 & 7.068 & 118295 & 1.1 & 2H-Pyran-2,6(3H)-dione \\
\hline 5 & 8.702 & 423879 & 3.93 & Glycine, N-(trifluoroacetyl)-, 1-methylpentyl ester \\
\hline 6 & 9.496 & 150019 & 1.39 & 1,5-anhydro-6-deoxyhexo-2,3-diulose \\
\hline 7 & 10.032 & 51040 & 0.47 & 1-Heptanol, 6-methyl- \\
\hline 8 & 10.97 & 1547557 & 14.35 & 5-Hydroxymethylfurfural \\
\hline 9 & 11.793 & 140443 & 1.3 & 4H-Pyran-4-one, 2,3-dihydro-3,5-dihydroxy-6-methyl- \\
\hline 10 & 12.877 & 97534 & 0.9 & 1-Tridecanol \\
\hline 11 & 13.394 & 118862 & 1.1 & Bicyclo[7.2.0]undec-4-ene, 4,11,11-trimethyl-8 \\
\hline 12 & 13.864 & 739740 & 6.86 & 1,8-Nonadien-3-ol \\
\hline 13 & 14.102 & 66317 & 0.61 & Benzene, 1-(1,5-dimethyl-4-hexenyl)-4-methy \\
\hline 14 & 14.272 & 124985 & 1.16 & (1S,5S)-2-Methyl-5-((R)-6-methylhept-5-en-2-yl)bicyclo[3.1 \\
\hline 15 & 14.439 & 87835 & 0.81 & Phenol, 3,5-bis(1,1-dimethylethyl)- \\
\hline 16 & 14.65 & 139087 & 1.29 & (1R,5R)-4-Methylene-1-((R)-6-methylhept-5-en-2-yl)bicycle \\
\hline 17 & 15.313 & 26808 & 0.25 & 1-Isopropylbenzocyclobuten-1-ol \\
\hline 18 & 15.403 & 107623 & 1 & Pentafluoropropionic acid, undecyl ester \\
\hline 19 & 15.953 & 29755 & 0.28 & 2,6,10-Dodecatrien-1-ol, 3,7,11-trimethyl- \\
\hline 20 & 16.007 & 40408 & 0.37 & 2-Oxoadamantane-1-carboxamide, N-(1-phenylethyl)- \\
\hline 21 & 16.1 & 46858 & 0.43 & 1-Methyl-1-isopropoxy-1-silacyclohexane \\
\hline 22 & 16.319 & 503249 & 4.67 & ar-Turmerone \\
\hline 23 & 16.378 & 464133 & 4.3 & Tumerone \\
\hline 24 & 16.764 & 424480 & 3.94 & Curlone \\
\hline 25 & 17.664 & 63489 & 0.59 & 1-Hexadecanol \\
\hline 26 & 19.426 & 675715 & 6.27 & n-Hexadecanoic acid \\
\hline 27 & 19.737 & 266947 & 2.48 & Oxalic acid, monoamide, N-(3,4-dimethylphenyl)-, heptyl est \\
\hline 28 & 21.125 & 444757 & 4.12 & cis-9-Hexadecenal \\
\hline 29 & 21.326 & 233163 & 2.16 & Octadecanoic acid \\
\hline 30 & 21.569 & 91559 & 0.85 & (Z)-Ethyl heptadec-9-enoate \\
\hline 31 & 21.911 & 49358 & 0.46 & Unknown compound \\
\hline 32 & 25.279 & 151135 & 1.4 & 1,2-benzenedicarboxylic acid, diisooctyl es \\
\hline 33 & 27.929 & 196112 & 1.82 & 9-octadecenamide \\
\hline \multirow[t]{2}{*}{34} & 28.226 & 29515 & 0.27 & (3,7-dimethyl-octa-2,6-dienylsulfanyl)-benz \\
\hline & & 10784583 & 100 & \\
\hline
\end{tabular}

Table 2: GC-MS peak report of SCOBY extract (SE)

\begin{tabular}{lllll}
\hline Peak\# & R.Time & Area & Area\% & Compounds of SE \\
\hline 1 & 4.851 & 193897 & 1.89 & 2,4-dihydroxy-2,5-dimethyl-3(2h)-furan-3-one \\
2 & 5.32 & 200609 & 1.96 & 1,4-diazabicyclo[2.2.2]octane \\
3 & 6.037 & 74123 & 0.72 & 1-propanol, 2-methyl-2-[(2-methyl-2-propenyl)oxy]- \\
4 & 6.991 & 418123 & 4.08 & Thymine \\
5 & 7.777 & 1223451 & 11.93 & 4H-pyran-4-one, 2,3-dihydro-3,5-dihydroxy-6-methyl- \\
6 & 8.807 & 214122 & 2.09 & 1,3-Dioxolane, 4-methylene-
\end{tabular}




\begin{tabular}{lllll}
7 & 9.379 & 2324289 & 22.66 & 5-Hydroxymethylfurfural \\
8 & 10.265 & 415246 & 4.05 & 2,4(3H,5H)-furandione, 3-propyl- \\
9 & 11.094 & 559322 & 5.45 & Isovaleric acid \\
10 & 12.59 & 1231914 & 12.01 & Guanosine \\
11 & 13.907 & 1821421 & 17.75 & Hexadecane \\
12 & 14.951 & 89934 & 0.88 & 1,6-methanonaphthalen-1(2h)-ol, octahydro-4,8a,9,9-tetramethyl-, \\
13 & 16.859 & 892196 & 8.7 & Caffeine \\
14 & 17.788 & 448012 & 4.37 & Dibutyl phthalate \\
15 & 23.296 & 152277 & 1.48 & 1,2-Benzenedicarboxylic acid \\
\hline
\end{tabular}

amount of caffeine from tea infusion. Furthermore, this should be left over future microbiological and metabolic pathway studies on SCOBY.

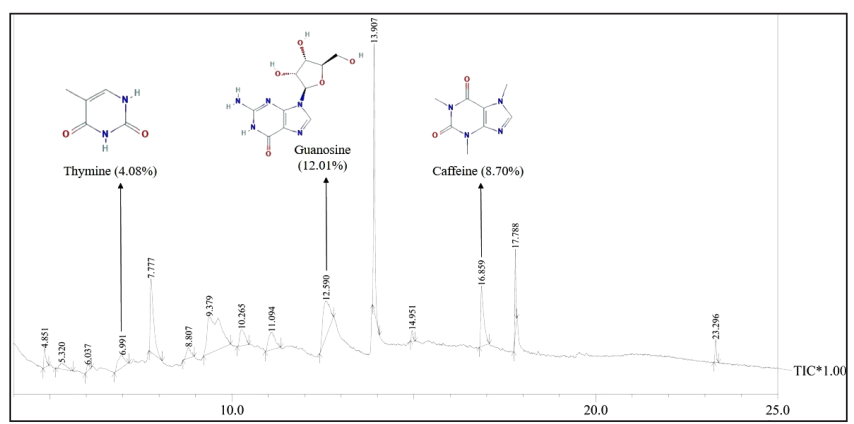

Fig. 3. Chromatogram of SCOBY extract showing peaks of caffeine, guanosine and thymine

\section{CONCLUSION}

Biochemistry of kombucha has already been well explored by various scientific communities where SCOBY extract remained untouched in the field of metabolomics. Removal of caffeine and other alkaloids have been explored by this study and through which we have come into a simple but effective conclusion that SCOBY of kombucha, not only helps in fermentation but also carry on some unexpected metabolic processes where it takes up all the stimulants and unpleasant compounds from kombucha to make the beverage completely decaffeinated and healthy as well. Microbiological experiments and higher analytical chemistry should be implemented along with this study to isolate stimulants like caffeine from old, discarded, unusable or molded SCOBY in future.

\section{REFERENCES}

Bhattacharya, M., Mandal, P.and Sen, A. 2009. In vitro detection of antioxidants in different solvent fractions of Ginger (Zingiber officinale Rosc.). Indian Journal of Plant Physiology, 14(1): 23-7.

Fontana, J.D., Franco, V.C., De Souza, S.J., Lyra, I.N. and De Souza, A.M. 1991. Nature of plant stimulators in the production of Acetobacter xylinum ("tea fungus") biofilm used in skin therapy. Applied Biochemistry and Biotechnology, 28(1): 341-51.

Greenwalt, C.J., Steinkraus, K.H. and Ledford, R.A. 2000. Kombucha, the fermented tea: microbiology, composition, and claimed health effects. Journal of Food Protection, 63(7): 976-81.

Jaber-Vazdekis, N.E., Gutierrez-Nicolás, F., Ravelo, Á.G. and Zárate, R. 2006. Studies on tropane alkaloid extraction by volatile organic solvents: dichloromethane vs. chloroform, Phytochemical Analysis: An International Journal of Plant Chemical and Biochemical Techniques, 17(2): 107-13.

Licker, J.L., Acree, T.E. and Henick-Kling, T. 1998. What is" Brett"(Brettanomyces) flavor?: A preliminary investigation, https://doi.org/10.1021/bk-1998-0714.ch008

Majumder, S., Ghosh, A. and Bhattacharya, M. 2020. Natural anti-inflammatory terpenoids in Camellia japonica leaf and probable biosynthesis pathways of the metabolome. Bulletin of the National Research Centre, 44(1): 1-4.

Malbaša, R., Lončar, E., Djurić, M., Klašnja, M., Kolarov, L.J. and Markov, S. 2006. Scale-up of black tea batch fermentation by kombucha. Food and Bioproducts Processing, 84(3): 193-9.

Negishi, O., Ozawa, T. and Imagawa, H. 1992. Biosynthesis of caffeine from purine nucleotides in tea plant. Bioscience, Biotechnology, and Biochemistry, 56(3): 499-503.

Santra, S.C., Chatterjee, T.P. and Das, A.P. 2006. College Botany Practical. Vol. 1. New Central Book Agency (P) Ltd. pp. 221.

Shalmashi, A. and Golmohammad, F. 2010. Solubility of caffeine in water, ethyl acetate, ethanol, carbon 
tetrachloride, methanol, chloroform, dichloromethane, and acetone between 298 and 323 K. Latin American Applied Research, 40(3): 283.

Šormová, Z., Melichar, O. and Šorm, F. 1960. Some pyrimidine derivatives as new types of plant stimulants. Collection of Czechoslovak Chemical Communications, 25(11): 2889-98.
Zhu, C., Li, F., Zhou, X., Lin, L. and Zhang, T. 2014. Kombucha-synthesized bacterial cellulose: Preparation, characterization, and biocompatibility evaluation. Journal of Biomedical Materials Research Part A, 102(5): 1548-57. 